TUBERCULOSIS

\title{
Transmission of tuberculosis from smear negative patients: a molecular epidemiology study
}

\author{
E Hernández-Garduño, V Cook, D Kunimoto, R K Elwood, W A Black, J M FitzGerald
}

Thorax 2004;59:286-290. doi: 10.1136/thx.2003.011759

See end of article for authors' affiliations

....................

Correspondence to: Professor J M FitzGerald

Center for Clinical

Epidemiology and

Evaluation, Vancouver

General Hospital, VGH

Research Pavilion, 828

West 10th Avenue

Vancouver, BC V5Z1L8;

markf@interchange.ubc.ca

Received 18 June 2003

Accepted

30 December 2003

\begin{abstract}
Background: While smear positive patients with tuberculosis (TB) are considered more infectious than smear negative patients, the latter can also transmit TB.

Methods: In a molecular epidemiology study of 791 patients in the Greater Vancouver regional district, the number of episodes of TB transmission from two groups of smear negative clustered patients by RFLP (assumed to be involved in recent transmission) was estimated after assessing for potential bias. Group 1 $(\mathrm{n}=79)$ included patients with pulmonary TB or pulmonary + extrapulmonary disease (PTB or PTB+EPTB); group $2(n=129)$ included all patients in group 1 + extrapulmonary cases alone.

Results: In the total sample the mean (SD) age was 51 (21) years, $54.3 \%$ were male, and $17.0 \%$ of patients were clustered. Compared with smear negative patients, smear positive patients were more likely to be in a cluster $(O R=2.0,95 \% \mathrm{Cl} 1.1$ to 3.6$)$ and to have had a history of ethanol abuse $(O R=2.7,95 \%$ $\mathrm{Cl} 1.0$ to 6.7$)$, diabetes mellitus $(\mathrm{OR}=2.8,95 \% \mathrm{Cl} 1.1$ to 7.0$)$, injection drug use $(\mathrm{OR}=3.1,95 \% \mathrm{Cl} 1.1$ to 8.3), and to have had a previous hospital admission (OR=8.5, 95\% Cl 5.1 to 14.0). The proportion of episodes of transmission from smear negative clustered patients ranged from $17.3 \%$ to $22.2 \%$ in group 1 and from $25 \%$ to $41 \%$ in group 2 .

Conclusion: In Greater Vancouver, smear negative cases appear responsible for at least one sixth of culture positive episodes of TB transmission.
\end{abstract}

1 has been estimated that 5000-10 000 acid fast bacilli (AFB) must be present in sputum for detection by smear testing, whereas only 10-100 viable organisms are required for a sputum culture to be positive. ${ }^{12}$ Molecular epidemiology has given us new insights into the transmission of tuberculosis (TB)..$^{3-5} \mathrm{~TB}$ control programmes place greatest emphasis on the early diagnosis of smear positive pulmonary TB (PTB) to ensure prompt initiation of treatment to reduce the transmission of infection. ${ }^{6}$ Mycobacterium tuberculosis is predominantly spread by patients with PTB, and it is widely believed that smear negative cases are less infectious than smear positive cases. Historically, studies have shown contacts of smear positive cases to be more likely to have a positive skin test result than smear negative cases..$^{78}$ However, there are limited data on the transmission of infection from smear negative, culture positive cases using molecular epidemiological techniques.

A study from San Francisco ${ }^{9}$ showed that at least $17 \%$ of TB cases were the result of transmission from smear negative cases with TB. However, this study was limited because the index cases with extrapulmonary TB (EPTB) without pulmonary investigation (sputum smears) were excluded, reducing the number of clusters. In this study we have estimated the number of smear negative transmission events in clustered patients with pulmonary involvement. Patients with EPTB, who represent $27 \%$ of clustered cases in Vancouver, ${ }^{10}$ were also included as they have been shown to be a possible source for an outbreak of $\mathrm{TB}^{1{ }^{11}}{ }^{12} \mathrm{We}$ hypothesised that the analysis of TB transmission in patients with extrapulmonary disease could have considerable impact on infection control practice.

\section{METHODS}

In this population based study all patients from the Greater Vancouver regional district with culture positive for $M$ tuberculosis reported to the Division of Tuberculosis Control of the BC Centre for Disease Control from January 1995 to
March 1999 were studied. All mycobacteriological testing in British Columbia is performed at the BC Centre for Disease Control Laboratory Services. The standard restriction fragment length polymorphism (RFLP) technique was used to subtype $M$ tuberculosis using the IS6110 insertion sequence and has been described elsewhere. ${ }^{10}$ TB isolates were considered clustered if they shared an identical DNA fingerprint pattern. A cluster was defined as all patients having isolates with the same DNA fingerprint and was likely to be involved in recent transmission. A spoligotyping kit (Isogen Bioscience, Maarssen) was used as a secondary typing technique for strains with five copies or less of IS6110. ${ }^{13}$ Smear specimens were stained with Auramine O and confirmed by Ziehl Neelsen staining.

The results were collected from the computerised database maintained by the Division of TB Control. For each case of culture positive $\mathrm{TB}$ the results of the first three sputum smears were obtained. Patients were allocated into three groups:

- smear positive: at least one positive sputum smear;

- smear negative: three or more negative sputum smears;

- smear indeterminate: less than three sputum smears collected and, of those available, all were negative.

Cases were excluded if they met the standard criteria for laboratory cross contamination. ${ }^{14}$

In all patients the demographic and clinical characteristics-including age, sex, place of birth, HIV status, history of AIDS, BCG, ethanol abuse, diabetes mellitus, injection drug use, prior $\mathrm{TB}$, prior hospital admission, site of disease and tuberculin skin test result-were compared by smear status. Because the index case in some clusters had EPTB alone and therefore could be potentially infectious, ${ }^{11}{ }^{12}$ we determined the proportion of smear negative transmission events and the relative transmission risk in two groups of clustered cases according to the site of disease (table 1). 
Table 1 Division of study subjects into two groups according to site of disease

\begin{tabular}{ll}
\hline Group 1 & Group 2 \\
\hline Pulmonary TB (PTB) & Pulmonary TB \\
Pulmonary TB + extrapulmonary & Pulmonary TB + \\
TB (PTB + EPTB) & extrapulmonary TB \\
& EPTB alone \\
\hline
\end{tabular}

PTB, pulmonary tuberculosis; EPTB, extrapulmonary tuberculosis.

Index cases with EPTB alone often do not have sputum smears available and were identified as smear negative if there was a recent normal chest radiograph. This assumption was based on published studies where only $4 \%$ of patients with PTB and a normal chest radiograph were smear positive, $^{15}$ and $1.7-3 \%$ of patients with EPTB were smear positive in a setting where sputum smears were collected routinely in all patients with EPTB. ${ }^{16}$

A smear negative transmission event was defined as a single clustered patient who followed a smear negative case within the cluster and therefore was assumed to be infected by that smear negative case. For example, in a cluster of size three with a smear positive index case and two smear negative secondary cases there was one smear negative transmission event (table 2).

The index case was defined as the first patient in the cluster according to the date of diagnosis and all secondary cases were sorted chronologically. Misclassification of the index case may have occurred using this method instead of duration of symptoms. For example, if an index case of a cluster of size two was diagnosed in April with symptoms since February and the second patient was diagnosed in May with symptoms since January, this second patient could be considered the index case. To account for this potential bias, physician dictation notes and patients' records were reviewed to determine the date of onset of symptoms. Symptom onset within all clusters corresponded chronologically to the date of diagnosis, suggesting the date of diagnosis as classification.

Because the definition of the first patient in a cluster by onset of symptoms is variable and dependent on patient recall, the proportion of smear negative transmission events was also determined after excluding those clusters where the first two patients were diagnosed less than 5 months apart. In clusters of size greater than two, all secondary cases could have been infected by a smear positive index case and not the preceding smear negative case (second patient in the table 2). For this reason, we also estimated the proportion of smear negative transmission events in only clusters of size two.

A separate analysis was performed in Aboriginal Canadians as they were more likely to be clustered than foreign born or non-Aboriginal Canadian patients. ${ }^{10}$ Epidemiological linkages among clustered cases were also reviewed through the TB Registry. The local Institutional Review Board approved the study.

Table 2 Interpretation of when transmission event was declined to have occurred in clusters containing two or more negative smears

\begin{tabular}{ll}
\hline Cluster of size three & $\begin{array}{l}\text { Events of smear } \\
\text { negative transmission }\end{array}$ \\
\hline 1st patient: smear positive & Zero events \\
2nd patient: smear negative & Zero events \\
3rd patient: smear negative & One event \\
\hline
\end{tabular}

\section{STATISTICAL ANALYSIS}

Descriptive statistics were computed using SAS (Statistical Analysis System, Cary, NC). $\chi^{2}$ or Fisher's exact tests were used in the univariate analysis to assess statistical differences between groups $(\mathrm{p}<0.05)$. Binominal proportions were computed for one way tables and estimates of transmission are presented with $95 \%$ confidence intervals (CI). The relative transmission rate was calculated as the proportion of smear negative events in smear negative patients divided by the proportion of smear positive events in smear positive patients.

\section{RESULTS}

During the study period (January 1995 to March 1999) 806 patients with culture positive $M$ tuberculosis were diagnosed in the Greater Vancouver regional district. Thirteen patients were excluded because of duplicate entries or missing information and two were excluded as their culture results were considered contaminated. The final sample therefore consisted of 791 patients of mean (SD) age 51 (21) years, $54.3 \%$ were male, and $17.0 \%$ were clustered.

Table 3 shows the distribution of cases by smear status and demographic and clinical variables. Two hundred and forty two patients $(31 \%)$ were smear positive, $146(18 \%)$ were smear negative, and 403 (51\%) were smear indeterminate. Eighty eight percent of smear positive patients had PTB and only $2 \%$ of cases with EPTB were smear positive. Most smear indeterminate patients had EPTB (55.6\%). Patients with indeterminate smears were more likely to be female than the other two groups $(p=0.003)$. There was no statistically significant difference between the three groups with regard to mean age, BCG history, country of birth, HIV status, and history of AIDS. Smear positive patients were more likely than smear negative patients to be clustered (OR $=2.0,95 \%$ CI 1.1 to 3.6) and to have had a history of ethanol abuse $(\mathrm{OR}=2.7,95 \%$ CI 1.0 to 6.7$)$, diabetes mellitus $(\mathrm{OR}=2.8$, $95 \%$ CI 1.1 to 7.0$)$, injecting drug use (OR $=3.1,95 \%$ CI 1.1 to 8.3 ), and previous hospital admissions ( OR $=8.5,95 \%$ CI 5.1 to 14.0). Compared with smear indeterminate patients, smear positive and smear negative patients were more likely to have had pulmonary disease $(\mathrm{p}<0.01)$ and a positive skin test $(p<0.01)$ and were less likely to have had extrapulmonary disease $(p<0.01$, table 3$)$. There was no statistically significant difference between the three groups in Aboriginal Canadians (data not shown), except that smear positive Aboriginals were more likely to have had a history of hospital admission than the smear indeterminate and smear negative Aboriginals together (OR 5.0, 95\% CI 1.0 to 25.4).

\section{Analysis of clusters}

Group 1 (patients with PTB or PTB+EPTB)

There were 79 clustered patients in 27 clusters. In three clusters all patients were smear negative. The mean (SD) age was 47.5 (19) years (range 4-96) and 63\% were male.

Of the 27 clusters, six (22.2\%) had a smear negative index case and nine (17.3\%) out of 52 secondary cases (95\% CI 10 to 27) were attributed to smear negative transmission in clusters with a smear negative index case (table 4). A total of $15(19 \%)$ smear negative patients transmitted the disease to the next patient in the cluster (95\% CI 11 to 29 ) regardless of the smear status of the index case ("all clusters" in fig 1). There were 20 clusters of size two with four index cases (20\%) identified as smear negative (95\% CI 2 to 23 ). There were 13 clusters with 31 patients where the first two patients were diagnosed more than 5 months apart with a total of six (19.3\%) events of smear negative transmission (95\% CI 7 to 37 , fig 1). The relative transmission rate in group 1 was 0.21 ( $95 \%$ CI 0.09 to 0.38 , table 4 ). Although not significant, HIV positive index cases were more likely to be smear positive 
Table 3 Demographic and clinical characteristics of patients by smear status, $\mathrm{n}(\%)$

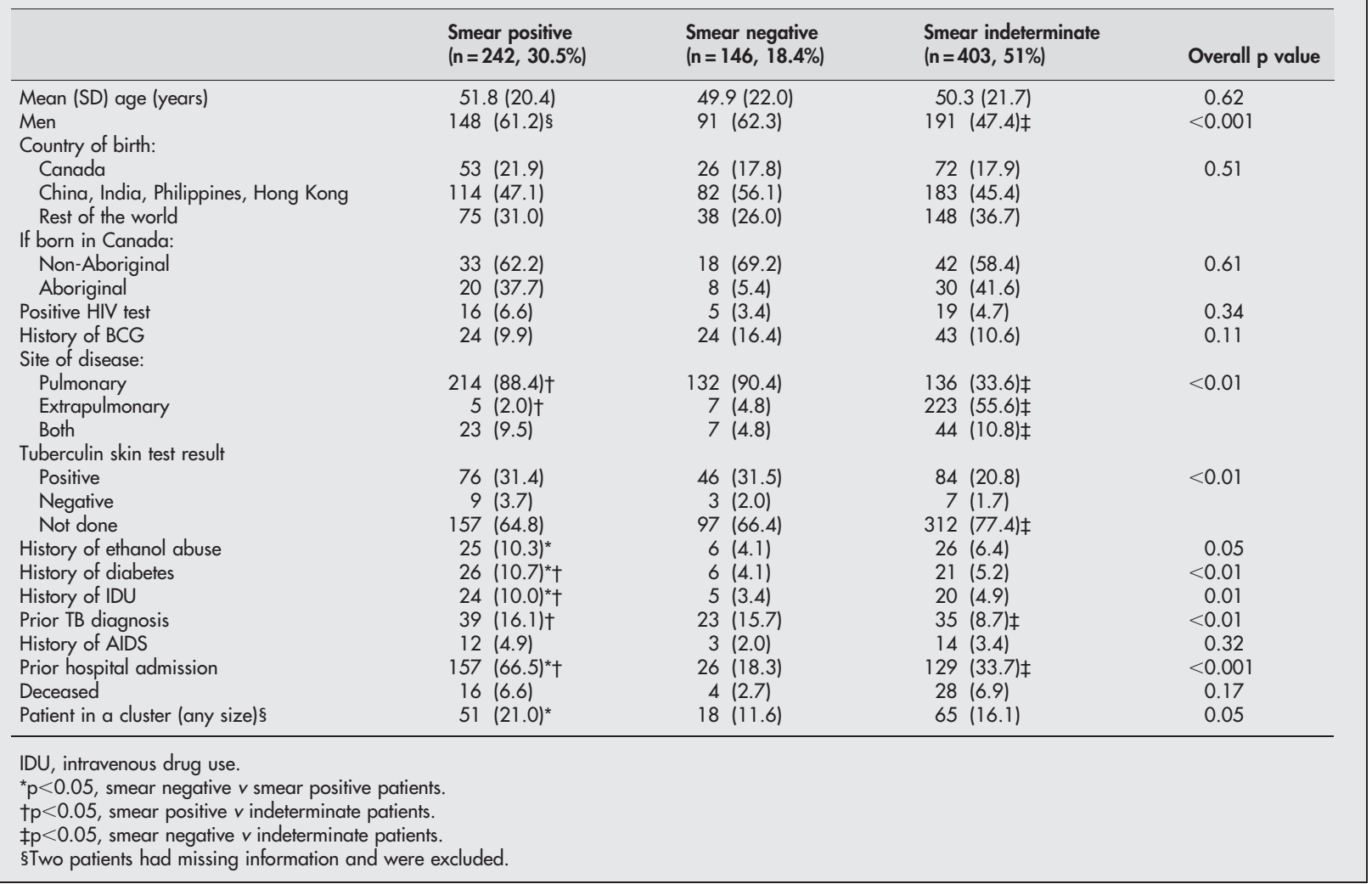

than smear negative index cases with a positive HIV result $(4 / 21(19 \%) \vee \mathrm{l} / 6(17 \%), \mathrm{p}=0.69)$ in this group.

Using traditional contact tracing methods, a total of 10 patients were found to be epidemiologicaly linked within a given cluster $(12.6 \%)$. These included two clusters of size two (both index cases were smear positive), one cluster of size three (the index case was smear positive and knew the third patient), one cluster of size four (the first two patients in this cluster knew each other and were smear negative), and two patients were in contact with each other in the largest cluster of 13 (the fifth and sixth patients, smear positive and negative, respectively). There were two events of smear negative transmission $(20 \%)$ in this group of 10 epidemiologicaly linked patients. Four clusters included only

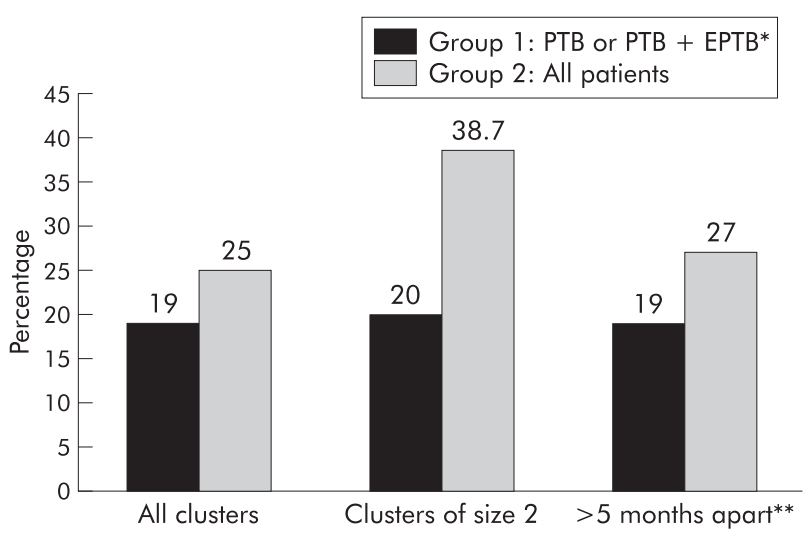

Figure 1 Proportion of smear negative transmission events in different subgroups by site of disease. ${ }^{*} \mathrm{PTB}=$ pulmonary tuberculosis; $\mathrm{EPTB}=$ extrapulmonary tuberculosis. ${ }^{*}$ Only clusters where the first two patients were diagnosed more than 5 months apart.
Aboriginal patients and the index case was smear negative in one cluster $(25 \%)$.

\section{Group 2 (all patients)}

After including patients with EPTB alone we identified 129 patients in 44 clusters (table 4 ). Nine of the index cases were smear indeterminate with EPTB only. The majority of chest radiographs were normal $(n=7)$, one chest radiograph showed fibronodular disease in the right upper lobe, and one case was diagnosed at necropsy without sputum or chest radiograph available. The patients with normal chest radiographs were assumed to be smear negative and the rest to be smear positive. Based on these assumptions, $18(41 \%)$ of the 44 clusters had a smear negative index case (table 4 ). The total number of secondary cases that could be attributed to smear negative transmission was $22(26 \%)$ of 85 (95\% CI 15 to 36$)$ with a relative transmission rate of 0.34 (95\% CI 0.21 to 0.49 , table 4 ). Thirty eight percent of the 31 clusters of size two had a smear negative index case (fig 1). The analysis by date of diagnosis showed that 16 of 59 patients $(27 \%, 95 \%$ CI 16 to 38 ) were infected by a smear negative case if the first two patients were diagnosed more than 5 months apart (fig 1). There were seven clusters that included only Aboriginal patients and the index case was smear negative in three $(43 \%)$.

\section{DISCUSSION}

In this study we have analysed the proportion of smear negative transmission events in two groups of clustered patients. In patients with PTB with or without EPTB (group 1) the proportion ranged from $17.3 \%$ to $22.2 \%$, while in patients with $\mathrm{TB}$ at any site (group 2) the proportion ranged from $25 \%$ to $41 \%$. Our estimate of smear negative episodes in clusters initiated by smear negative index cases in patients with pulmonary involvement $(0.21)$ was similar to that reported in San Francisco (0.22) for the minimum 
Table 4 Characteristics of clustered cases by site of TB

\begin{tabular}{|c|c|c|}
\hline & $\begin{array}{l}\text { Group } 1 \\
\text { (PTB or PTB+EPTB) }\end{array}$ & $\begin{array}{l}\text { Group } 2 \\
\text { (all patients) }\end{array}$ \\
\hline $\begin{array}{l}\text { No. of clusters } \\
\text { Total clustered patients } \\
\text { Total secondary cases } \\
\text { No. of clusters with smear negative } \\
\text { index cases } \\
\text { No. of secondary cases attributed to } \\
\text { smear negative transmission* } \\
\text { Relative transmission rate† }\end{array}$ & $\begin{array}{l}27 \\
79 \\
52 \\
6 / 27(22.2 \%) \\
(95 \% \mathrm{Cl} 12 \text { to } 31) \\
9 / 52(17.3 \%) \\
(95 \% \mathrm{Cl} 10 \text { to } 27) \\
0.21 \\
(95 \% \mathrm{Cl} 0.09 \text { to } 0.38)\end{array}$ & $\begin{array}{l}44 \\
129 \\
85 \\
18 / 44(41 \%) \\
(95 \% \mathrm{Cl} 31 \text { to } 52) \\
22 / 85(26 \%) \\
(95 \% \mathrm{Cl} 15 \text { to } 36) \\
0.34 \\
(95 \% \mathrm{Cl} 0.21 \text { to } 0.49)\end{array}$ \\
\hline
\end{tabular}

transmission rate of smear negative patients, ${ }^{9}$ which suggests that our estimate is accurate.

We determined that the date of diagnosis was consistent with the symptom onset, suggesting that the date of diagnosis is a valid method for identifying the index cases in clusters. Further analysis was performed after the exclusion of clusters where the first two patients were diagnosed less than 5 months apart to confirm that the estimates were similar (19\%), indicating that a reversed order of diagnosis bias was unlikely in our study population.

In the study from San Francisco the authors excluded those clusters where the first case had extrapulmonary disease but did not have pulmonary investigation as they were deemed to be non-infectious. In our analysis we included cases with extrapulmonary disease because they are involved in clusters and represent $27 \%$ of the clustered cases in the Greater Vancouver regional district. ${ }^{10}$ If clusters are excluded for any reason, the study of transmission is incomplete and may underestimate the TB transmission patterns in clustered cases as other routes of transmission. ${ }^{12}$

Higher estimates of transmission were found when patients with EPTB alone were included (range 25-41\%) than in patients with pulmonary disease. The higher proportion of smear negative episodes in this group may be overestimated as it was assumed that index cases with EPTB and a normal chest radiograph were negative. However, this assumption was based on published data where only $4 \%$ of patients with PTB and a normal chest radiograph were smear positive. ${ }^{15}$ Also, it is often local practice-with some exceptions ${ }^{16}$ - that sputum samples are not requested by physicians for patients with extrapulmonary disease and a normal chest radiograph.

A higher transmission rate when patients with EPTB are included also suggests that the infectiousness of patients with EPTB has previously been underestimated, and this is highlighted by the EPTB clustered index cases. For example, an outbreak of tuberculosis initially reported in North Dakota $^{11}$ identified the source case as a 9 year old child with cavitary disease felt to be secondary to endogenous reactivation. However, the index patient was an adult with extrapulmonary disease who was the female guardian of the child and had TB of the hip and a pelvic abscess. This index case had a positive tuberculin skin test result and a normal chest radiograph so, presumably because there was no clinical evidence of pulmonary disease, she was not considered to be infectious.

In a second example a patient with genitourinary $\mathrm{TB}$ was the index case for transmission of TB to healthcare workers in Middle Tennessee..$^{12}$ A total of 128 healthcare workers were exposed to this index case with no evidence of active PTB and $13 \%$ of the exposed workers had newly positive tuberculin skin tests. The irrigation or packing of the surgical site was associated with the conversion of the tuberculin skin tests among nurses. These examples confirm transmission of TB from patients with EPTB and implicate the need to include EPTB data in the analysis of smear negative transmitters. A recent study from Brazil showed that the yield of sputum cultures obtained by sputum induction was high in patients suspected of having pleural TB even when there was no radiographic evidence of pulmonary parenchymal disease. ${ }^{17}$

With the inclusion of patients with EPTB alone the transmission rate rose to 0.34 , which is similar to the rate of 0.28 reported in a study from Saskatchewan ${ }^{8}$ but lower than that found in Spain (0.47). ${ }^{18}$ This suggests that the smear negative transmission rates vary in different settings.

The analysis of clusters of size two is a particularly interesting novel approach because, if we assume that these clusters do not grow over time, this should provide a more accurate estimation than larger clusters. One limitation of analysing large clusters is the confusion caused by secondary cases with positive smears occurring within the cluster. These could potentially transmit the disease to subsequent patients and overestimate the proportion of smear negative episodes, even when the cluster is initiated by a smear negative index case. Another factor that could overestimate the proportion of smear negative transmission is the presence of false negative results due to reader inexperience, inadequate staining, or inspection of too few fields (a minimum of 100 fields requires inspection which can take $5-10$ minutes). ${ }^{19}$ This is unlikely to have been a factor in our study as all the mycobacteriological investigations were carried out in a single reference laboratory.

A potential bias considered in the San Francisco study was defined as the "missing source case bias". In our study all isolates of patients with TB in the Greater Vancouver regional district during the study period were fingerprinted, so the assessment for this bias was unnecessary. From a practical point of view, public health administrators can only make programme and management decisions based on the cases actually diagnosed rather than considering a group of possible undiagnosed cases. The current study, which included all cases diagnosed in the region over a 4 year period, is less likely than others to be biased by a failure to account for all cases.

There was a greater proportion of positive HIV tests in smear positive than in smear negative patients $(6.6 \% v 3.4 \%)$, although the difference was not statistically significant. A similar trend was observed in the HIV status of clustered index cases. These results are consistent with reports from North America ${ }^{20}$ but are contrary to those from African countries where an increased prevalence of smear negative $\mathrm{TB}$ in HIV infected patients has been reported. ${ }^{21-24} \mathrm{~A}$ study 
from Santo Domingo suggested that HIV positive individuals with TB are less likely than HIV negative individuals with TB to transmit TB to their close contacts. ${ }^{25}$ These studies suggest that transmission from smear negative HIV positive patients varies among populations and settings and remains a topic of interest.

A separate analysis in clusters including only Aboriginal patients was carried out to account for the higher rates of clustering in Aboriginal Canadians. This analysis showed higher estimates of smear negative transmission events compared with all clusters $(25 \% \vee 19 \%$ in the first group; $48 \% \vee 23 \%$ in the second group).

Even though the sample was small $(\mathrm{n}=10)$, we found that epidemiologically linked patients had similar estimates of smear negative transmission events $(20 \%)$ compared with all clusters, clusters of size two, and clusters where the first two patients were diagnosed more than 5 months apart (19\%, $20 \%$, and $19 \%$, respectively), which again suggests that the estimates are accurate.

The results of this study suggest that smear negative patients with $\mathrm{TB}$ are a significant source of disease transmission in at least $17 \%$ of the transmission events in the Greater Vancouver regional district. From a public health point of view, the focus of TB control has been the prompt diagnosis and management of smear positive TB cases. These findings suggest that it is important to establish the smear status of patients with extrapulmonary disease through sputum induction or other methods, particularly when the case is a putative index case.

These data suggest that patients with smear negative disease are the cause of transmission for at least one in six patients with pulmonary involvement. When patients with EPTB are included, there is a further increased risk of spread. It is unclear if this is from the site of extrapulmonary disease or unrecognised pulmonary involvement.

\section{ACKNOWLEDGEMENTS}

The authors thank the nurses, outreach workers, and laboratory technicians who facilitated the collection of epidemiological data and processing of samples for this study.

\section{Authors' affiliations}

V Cook, R K Elwood, W A Black, J M FitzGerald, Department of Medicine, The University of British Columbia, Vancouver, BC, Canada D Kunimoto, Department of Medicine, The University of Alberta, Edmonton, Alberta, Canada

E Hernández-Garduño, V Cook, R K Elwood, W A Black, British Columbia Center for Disease Control, Vancouver, BC, Canada J M FitzGerald, Center for Clinical Epidemiology and Evaluation, Vancouver General Hospital, Vancouver, BC, Canada

This study was funded by the Medical Services Branch of Health Canada and the BC Lung Association. Dr FitzGerald is a research scientist at the Vancouver Hospital and the recipient of a Canadian Institute for Health Research/BC Lung Investigator Award.

\section{REFERENCES}

1 Hobby GL, Holman AP, Iseman MD, et al. Enumeration of tubercle bacilli in sputum of patients with pulmonary tuberculosis. Antimicrob Agents Chemother 1973;4:94-104.

2 Yeager HJ Jr, Lacy J, Smith L, et al. Quantitative studies of mycobacterial populations in sputum and saliva. Am Rev Respir Dis 1967:95:998-1004.

3 Alland D, Kalkut GE, Moss AR, et al. Transmission of tuberculosis in New York City. An analysis by DNA fingerprinting and conventional epidemiology methods. N Engl J Med 1994;330:1710-6.

4 Small PM, Hopewell PC, Singh SP, et al. The epidemiology of tuberculosis in San Francisco. A population-based study using conventional and molecular methods. N Engl J Med 1994;330:1703-9.

5 Jasmer RM, Hahn JA, Small PM, et al. A molecular epidemiologic analysis of tuberculosis trends in San Francisco 1991-1997. Ann Intern Med 1999:30:971-8.

6 American Thoracic Society/Centers for Disease Control and Prevention/ Infectious Diseases Society of America. Treatment of tuberculosis. Am J Respir Crit Care Med 2003; 167:603-62.

7 Van Geuns HA, Meijer J, Styblo K. Results of contact examination in Rotterdam, 1967-69. Bull Int Union Tuberc 1975;50:107-19.

8 Grzybowsky S, Barnett GD, Styblo K. Contacts of cases of active pulmonary tuberculosis. Bull Int Union Tuberc 1975;50:90-106.

9 Behr MA, Warren SA, Salamon H, et al. Transmission of Mycobacterium tuberculosis from patients smear-negative for acid-fast bacilli. Lancet 1999;353:444-9

10 Hernández-Garduño E, Kunimoto D, Wang L, et al. Predictors of clustering of tuberculosis in Greater Vancouver: a molecular epidemiologic study. Can Med Assoc J 2002;167:349-52.

11 Curtis AB, Ridzon R, Vogel R, et al. Extensive transmission of Mycobacterium tuberculosis from a child. N Engl J Med 1999;341:1491-5.

12 D'Agata EM, Wise S, Stewart A, et al. Nosocomial transmission of Mycobacterium tuberculosis from an extrapulmonary site. Infect Control Hosp Epidemiol 2001;22:10-2.

13 van Soolingen D. Molecular epidemiology of tuberculosis and other mycobacterial infections: main methodologies and achievements. J Intern Med 2001;249:1-26.

14 Jasmer RM, Roemer M, Hamilton J, et al. A prospective, multicenter study of laboratory cross-contamination of Mycobacterium tuberculosis cultures. Emerg Infect Dis 2002;8:1260-3.

15 Marciniuk DD, McNab BD, Martin WT, et al. Detection of pulmonary tuberculosis in patients with a normal chest radiograph. Chest 1999; 115:445-52.

16 Kwanjana IH, Harries AD, Hargreaves NJ, et al. Sputum-smear examination in patients with extrapulmonary tuberculosis in Malawi. Trans $R$ Soc Trop Med Hyg 2000;94:395-8.

17 Conde MB, Loivos AC, Rezende VM, et al. Yield of sputum induction in the diagnosis of pleural tuberculosis. Am J Respir Crit Care Med 2003; 167:723-5

18 Vidal R, Mirvitlles M, Cayla JA, et al. A contagiousness study in 3071 familial contacts of tuberculosis patients. Med Clin Barc 1997;108:361-5.

19 International Union Against Tuberculosis and Lung Disease. Technical guide for sputum examination for tuberculosis by direct microscopy. 5th ed. Paris: International Union Against Tuberculosis and Lung Disease, 2000.

20 Smith RL, Yew K, Berkowitz KA, et al. Factors affecting the yield of acid-fast sputum smears in patients with HIV and tuberculosis. Chest 1994;106:684-6.

21 Karstaedt AS, Jones N, Khoosal M, et al. The bacteriology of pulmonary tuberculosis in a population with high human immunodeficiency virus seroprevalence. Int J Tuberc Lung Dis 1998;2:312-6.

22 Colebunders RL, Ryder RW, Nzilambi N, et al. HIV infection in patients with tuberculosis in Kinshasa, Zaire. Am Rev Respir Dis 1989;139:1082-5.

23 Nunn P, Mungai M, Nyamwaya J, et al. The effect of human immunodeficiency virus type-1 on the infectiousness of tuberculosis. Tuberc Lung Dis 1994;75:25-32.

24 Elliott AM, Namaambo K, Allen BW, et al. Negative sputum smear results in HIV-positive patients with pulmonary tuberculosis in Lusaka, Zambia. Tuberc Lung Dis 1993;74:191-4.

25 Espinal EA, Pérez EN, Báez J, et al. Infectiousness of Mycobacterium tuberculosis in HIV-1-infected patients with tuberculosis: a prospective study. Lancet 2000;355:275-80. 